\title{
Herramientas TIC y el aprendizaje en los estudiantes de derecho de una universidad privada de Trujillo 2021
}

\author{
Héctor Florencio Moreno Castillo \\ hmorenoc@ucvvirtual.edu.pe \\ Investigador independiente \\ Silvia Ana Valverde Zavaleta \\ savalverdez@ucvvirtual.edu.pe \\ Universidad César Vallejo \\ Trujillo - Perú
}

\section{RESUMEN}

La presente investigación titulada: "Herramientas TIC y el aprendizaje en los estudiantes de Derecho de una universidad privada en Trujillo 2021. Se consideró una población de 97 estudiantes de dicha institución. La metodología utilizada fue el enfoque cuantitativo, el nivel de investigación es descriptiva, el tipo de investigación es correlacional básica, se analiza y explica por medio de la descripción a nivel de las variables Tics y el Aprendizaje, tiene como diseño no experimental. La técnica para la recolección de datos fue a través de una encuesta por variable; el instrumento empleado fue el cuestionario y la escala de Linkert. Los resultados obtenidos en la investigación por medio de la prueba no probabilística de rho de Spearman aplicada a los estudiantes brindan como coeficiente de correlación 0,512 y una significancia $\mathrm{p}<$ de 0.05 lo cual indica una correlación directa, positiva y moderada. Así mismo, el $56.7 \%$ de los estudiantes indican un nivel excelente en cuanto al uso de las Tics. En cuanto a la variable Aprendizaje se percibe un nivel excelente con un valor de 76.3. Por lo tanto, mientras se incremente el campo de acción de la tecnología en docentes y alumnos, ambos reflejarán un mejor desempeño académico.

Palabras clave: TIC, aprendizaje, incremento cognitivo, desenvolvimiento. 


\title{
ICT tools and learning in law students from a private university in Trujillo 2021
}

\begin{abstract}
The present investigation titled: "ICT tools and learning in law students of a private university in Trujillo 2021. A population of 97 students of said institution was considered. The methodology used was the quantitative approach, the research level is descriptive, the type of research is basic correlational, it is analyzed and explained by means of the description at the level of the Tics and Learning variables, it has a non-experimental design. The technique for data collection was through a survey by variable; the instrument used was the questionnaire and the Linkert scale. The results obtained in the investigation by means of the non-probabilistic Spearman $\mathrm{R}$ test applied to the students provides a correlation coefficient of 0.512 and a significance $\mathrm{p}<0.05$ which indicates a direct, positive and moderate correlation. Likewise, $56.7 \%$ of the students indicate an excellent level in terms of the use of ICTs. Regarding the Learning variable, an excellent level is perceived with a value of 76.3. Therefore, while the field of action of technology in teachers and students increases, both will reflect a better academic performance.
\end{abstract}

Keywords: ICT, learning, cognitive growth, development.

Artículo recibido: 20 diciembre. 2021

Aceptado para publicación: 10 enero 2022 Correspondencia: hectormoreno2@outlook.com.pe Conflictos de Interés: Ninguna que declarar 


\section{INTRODUCCIÓN}

En el contexto internacional la realidad es diferente, desde la concepción de lo que enmarca educar que junto a los lineamientos a los que se arribe como conclusión se ven plasmados en un diseño curricular de manera integral. Como lo plantea Hermosa del Vasto (2015) al demostrar la incidencia de lasTics en el proceso de aprendizaje que fusionada a la enseñanza tradicional los resultados son más positivos para el estudiante, docente, entidad educativa y sociedad. La sociedad europea y americana en cada etapa del desarrollo educativo van introduciendo metodologías que se fusionan con la tecnología ya que en ese contexto constituye un elemento de vanguardia.

Así mismo, UNESCO (2017) describe objetivos y la necesidad imperiosa de ir incorporando la tecnología en los diferentes contextos educativos con miras al 2030. Esto quiere decir, que "la tecnología tiene un matiz de indispensable para salir de encrucijadas conceptuales o mecánicas a las que se enfrenten los estudiantes en los diferentes contextos”, como lo expresa Hinojo, (2019), ya que lo emplean como una especie de biblioteca, de la cual, pueden recuperar información para consultarla y analizarla.

Tomando en consideración University of Glasgow (2019) plantea indicadores que se agrupan en cinco áreas como: enseñanza (a la formación educativa -académica), investigación (en base a la persona ética), citaciones (relación con la investigación), perspectiva internacional (contexto personal, estudiantil e investigación), ingresos en la industria (aplicación del conocimiento).

En el contexto nacional, las concepciones plasmadas en los proyectos educativos emitidos por el ministerio de educación enmarcan aspectos ortodoxos, ya que es muy poco en lo que se refiere a innovación o empleo de la tecnología.

Según Patiño (2018) expresa en una entrevista, "los docentes deben estar en una especie de contacto virtual para guiar a los estudiantes en el logro del objetivo y su evolución académica y profesional"'.

Nuestra realidad educativa nacional, en tecnología se va incrementando como lo refleja el censo elaborado Instituto Nacional de Estadística e Informática (2018): 92,8\% tienen: celular o teléfono fijo o internet o TV por cable. De acuerdo al padre de familia: con primaria aumentó en 0,6\%; con secundaria, $0.3 \%$ Padres con educación universitaria (institutos) 
siguen igual al 2017. A su residencia, el 54,9\% de Lima Metropolitana cuentan con un computador; urbano, $41,7 \%$ y en rurales, $5,5 \%$; así como el $90,8 \%$ universitarios acceden a la tecnología y el 79,5\% no universitaria; secundaria 54,6\% y primaria o menor nivel, $17,6 \%$. A nivel regional, es muy caótico ya que gira en base a una educación tradicionalista y sin objetivos claros para formar seres integrales y esto puede ser percibido en los egresados de cada ciclo educativo pues no poseen infraestructura ni tecnología.

En el ámbito local, la manera en que se desarrolla la educación en la Universidad Privada Antenor Orrego refleja una manera tradicional en la que solo se sintetiza en el manejo cognitivo - memorístico mayormente, considerando la especialidad, generando cierta limitación en los estudiantes para enfrentar ciertas actividades académicas, ante esta realidad surge la necesidad de incrementar en todas las mallas curriculares temáticas tecnológicas. Ante esta realidad se plantea como problema general: ¿Qué relación hay entre herramientas TICS en el aprendizaje de Lenguaje de los estudiantes de la Escuela Profesional de Derecho y Ciencias Políticas de la Universidad Privada Antenor Orrego del distrito de Trujillo - La Libertad en el año 2021? Se plantea como objetivo General: Determinar la influencia de las herramientas TICS en el aprendizaje de Lenguaje de los estudiantes de la Escuela Profesional de Derecho y Ciencias Políticas de la Universidad Privada Antenor Orrego del distrito de Trujillo - La Libertad en el año 2021. Así mismo se plantea la hipótesis general: Existe relación entre las herramientas TICS en el aprendizaje de Lenguaje y refleja una influencia significativa en los estudiantes de la Escuela Profesional de Derecho y Ciencias Políticas de la Universidad Privada Antenor Orrego del distrito de Trujillo - La Libertad en el año 2021. La presente investigación se justifica en los siguientes criterios (Hernández, Fernández, \& Baptista, 2014).

Por su Conveniencia. El empleo de las herramientas tics en todos los contextos educativos no ha enmarcado una evolución, en el campo educativo y profesional, ya que no ha generado un desarrollo basado en la interacción durante el desarrollo social y académico a través del tiempo y en todos los espacios.

Por su Relevancia social: Al estar en un mundo globalizado el aporte que se deriva de este trabajo y de manera social es muy relevante al no haberse percibido la contribución al desarrollo individual, social, regional y nacional. 
Por sus Implicaciones prácticas: En este contexto se describe la incidencia de manera trascendente en el aspecto práctico educativo y social; de tal manera, que por medio de la interacción académica se procurará generar una estabilidad social y desprender el desarrollo de una sociedad o nación.

Por su Valor teórico: Todo descubrimiento científico es nutritivo ante futuras investigaciones; la presente investigación busca contribuir a esta realidad aportando cierto conocimiento o panorama que permita a los futuros estudiantes considerarla como una fuente de influencia académica para ir modificando el conocimiento a través del tiempo, ya es una realidad que desde tiempos atrás no se percibe.

Por su Utilidad metodológica: En la presente investigación se estructuró instrumentos que permitan recoger información para resaltar su importancia, todo este panorama estuvo enmarcado en el método científico, lo cual será el indicador que le dé validez a este trabajo de investigación y en la cual se justifica.

El sentido presente estudio se fundamentó en los siguientes antecedentes internacionales: Zambrano y Zambrano (2019) en su investigación titulada Las tecnologías de la información y las comunicaciones (TICs) en la educación superior expresa que "el empleo de las Tics en el contexto superior es de suma importancia en el contexto educativo y se convierte en una herramienta que posibilita la fusión de diferentes elementos, con sus diferencias que generan una ventaja para el estudiante". Posibilita la trasmisión de información en un tiempo corto de manera amena y variada; generado por un ambiente favorable para el aprendizaje ya que es de carácter interactivo, integrando contenidos y la armonía de eventos o acciones formativas en el contexto docente, de su empleabilidad o como investigador de diferentes tiempos y contextos.

Por su parte, Francis (2017) En su artículo científico The Effects Of Technology On Student Motivation And Engagement In Classroom-Based Learning aplicado a una población de 348 estudiantes a través del método de análisis de datos, expresa: La tecnología ha visto una creciente integración generalizada en la vida cotidiana, donde el acceso a vastas cantidades de información ahora está disponible con facilidad.(...) Para crear una efectiva aula del siglo XXI que satisfaga las necesidades del estudiante y su motivación para aprender y los efectos que la tecnología tiene en la educación inclusiva. Se puede inferir, que al estar dentro de un 
mundo globalizado los inventos y los avances de la ciencia no se detienen; todas las personas, sin considerar la edad, deben estar capacitados en el dominio de estrategias, métodos, procesos, teorías para que de una manera transversal contribuyan con el desarrollo cognitivo, convirtiéndose a través de la experiencia en un factor indispensable como lo es ahora la tecnología.

Según Briceño, Flores y Gómez (2019) en su artículo científico Usos de las Tics en preescolar: hacia la integración curricular. aplicado a cinco docentes y 25 estudiantes en conjunto con sus familiares, esta investigación enmarcó el estudio de casos de manera cualitativa, concluye que "el empleo de las Tics por parte de los docentes de preescolar está condicionada al interés de los niños, pero limitada por los programas de los que disponen o la conexión con la que cuentan. A pesar que las familias se vinculan al proceso formativos y el acompañamiento a los estudiantes es fundamental para el desarrollo de un plan curricular vinculante el cual refleje una mayor participación en la toma de decisiones para un currículo y el intercambio de experiencias de aprendizaje en los hogares sobre el uso de las Tics que incrementen el proceso de aprendizaje en los niños.

Por su parte, Vidal, Rui y Rocha (2019) en su trabajo de investigación titulada A systematic literature review on maturity models for information systems in higher education institutions. expresa: los modelos de madurez se han introducido en las últimas cinco décadas como guías y referencias para la gestión del sistema de información (SI) en organizaciones de diferentes sectores. En el sector educativo, los modelos de madurez también se han utilizado para evaluar las instituciones de educación superior (IES) en varias dimensiones, como la tecnología de la información y la comunicación (TIC), la gestión de procesos, los currículos de cursos, la acreditación de cursos /IES, e-/m -aprendizaje, cursos en línea y estrategias pedagógicas (...) del mismo modo lo plantea Islas (2017) en su artículo científico. Lo que nos comunica es que el uso de las Tics es muy importante en este mundo actual, pero el impartir este tipo de enseñanza por medio de estas técnicas o instrumentos no solo queda en lo teórico sino, más bien, se necesita de un acompañamiento pedagógico práctico para reforzar las fortalezas que se pueden percibir en los estudiantes y retroalimentarlas.

Según Basri, Alandejani y Almadani (2018) en el artículo científico Traducido ICT adoption Impact on students Academic Performance: Evidence from Saudi Universities. Concluye: un 
hallazgo interesante en este estudio afirma que ha habido un mayor uso de las Tics, en particular para buscar información relacionada con los estudios. Por lo tanto, la adopción de las Tics es muy alta entre los estudiantes universitarios, ya que la mayoría (más de dos tercios) usan las Tics al menos durante dos horas en un día. Ante esta realidad, es necesario ofrecer un apoyo total al acceso de los estudiantes a las Tics, ya que tiene el potencial de mejorar su rendimiento académico.

Respecto al panorama nacional: Según Cabrera (2019) en su tesis doctoral Entornos virtuales de aprendizaje mediante una nueva metodología aplicados a las competencias tecnológicas del Docentes expresa como conclusión que la aplicación de Entornos Virtuales enmarcada en la metodología denominada MI-EVA incrementó la cantidad de Docentes Universitarios de la Facultad de Derecho USMP valoran la aplicación de esta metodología en la enseñanza de sus estudiante, y aumenta la dimensión actitudinal en el desarrollo de la competencias en el uso de la tecnología por parte del docente. Así también lo plantea en su artículo científico Del mismo lo describe Valle (2018) en su artículo de investigación Sin las TIC, la educación superior y de posgrado no está completa. En Y. Valle, Sin las TIC, la educación superior y de posgrado no está completa lo que desea trasmitir este autor, con el método denominado MI-EVA (entornos virtuales de aprendizaje) en la realidad actual dentro del contexto educativo no se debe desarrollar una carrera profesional dentro de la universidad sin considerar la tecnología para el desarrollo de los educandos y, al mismo tiempo, enfatizar en los docentes.

Según Arias (2019) en su tesis doctoral Uso del Exelearning, aplicación de contenidos digitales y su relación con el proceso aprendizaje expresa que el uso de exelearning (herramienta de código abierto (open source) para la creación de esquemas o contenidos educativos y su relación con el proceso de aprendizaje en la Universidad Nacional José Faustino Sánchez Carrión 2017, fue positiva, ya que ante la aplicación de un pretest que arrojó cierto grado de conocimiento, el cual este se incrementó después del uso del exelearning por parte de los estudiantes y se ve reflejado en la aplicación de un postest. Según (Reyes, 2017) en su tesis doctoral Modelo de calidad aviso en la implementación de entornos Blended Learning para la mejora del proceso enseñanza - aprendizaje en la educación superior universitaria concluye: Luego de la aplicación del modelo de calidad en 
la implementación de entornos blended learning, se pudo observar que hubo una mejora en el proceso de enseñanza - aprendizaje, pues se redujo de manera significativa la cantidad de estudiantes desaprobados e inhabilitados, y el nivel de uso y satisfacción con la plataforma virtual de cada uno de los cursos también se elevó de manera importante, (...).

Según Gil (2002), define como herramientas tics al conjunto de aplicaciones, secuencias, técnicas, procedimientos o metodologías que se vinculan con las señales digitales analógicas, a la estructuración de textos, producción de imágenes las cuales pueden enmarcar diferentes contextos en tiempo real.

La teoría que fundamenta la variable está enmarcada en el Conductismo, siendo su autor Skiner: Esta teoría se basa de manera general en el estímulo y respuesta que tienen las personas ante un determinado evento o situación, modificando su panorama cognitivo y conductual, basado en un condicionamiento positivo; pero ésta realidad, en el mundo actual, se genera ante la interacción del mundo interno y la realidad tecnológica que rodea a las personas de todos los contextos vivenciales a través de un proceso de retroalimentación basado en el ejercicio y la práctica.

Por su parte, Ochoa y Cordero (2002), define como herramientas Tics al conjunto de procesos y productos enmarcadas en un equipo compuesto por un hardware y software (parte visible e invisible para generar campos de ayuda), con el que se puede generar formas para la trasmisión de información entre personas con esquemas similares de almacenaje, procesamiento y traslado de información dentro del campo digital.

Por otro lado, Para (Avila, 2016) la importancia de las herramientas Tics dentro de las actividades que desarrollan tanto docentes como estudiantes, desde el empleo de estas ambos entes pueden planificar, diseñar secuencias o instrumentos que serán empleados en las sesiones de clase (textos en Word y convertirlos en pdf), asimismo, incorporar videos, grabaciones u otras herramientas que desarrollen las competencias de adquisición y también considerar el aspecto de la investigación; afianzando los aspectos comunicativos entre docente y estudiantes a través de la interacción considerando las herramientas Tics.

García de Merlano (2013) expresa que la importancia de las herramientas Tics en el proceso enseñanza-aprendizaje giran en el propiciar espacios significativos en los estudiantes y al ser una ruta de análisis en cuanto a las acciones realizadas por los docentes; que deben enfocarse 
a la participación de todos los estudiantes en una visión y el planteamiento de objetivos primero a nivel institucional y, a continuación, marcar el derrotero para encontrar sentido a las acciones de los estudiantes.

Estos dos conceptos se fusionan con el pasar del tiempo en una especie de catarsis educativa debemos inspirar el uso de la tecnología y todo lo que encontramos en el mundo interactivo considerando la corriente del pensamiento denominada Empirismo de Locke y Hume que nos dicen, al ser una teoría filosófica gira en base a la experiencia y la evidencia, especialmente la percepción a través de los sentidos, es decir, la realidad natural nos plantea situaciones que se encaminan en ideas y éstas generan nuevo conocimiento, el cual con el tiempo se toma como referencia y es sujeta a modificación de acuerdo a la nueva vivencia. Considerando las variables del presente problema se plantea una fusión constante para generar la investigación y que nuestros estudiantes vayan desarrollando su panorama cognitivo y académico basado en sus vivencias y creatividad para ser más competentes en el mundo actual ya que estamos en un mundo de cambios y sobre todo globalizado.

\section{ESTRATEGIA METODOLÓGICAS}

El enfoque sobre el cual girará el presente proyecto es cualitativo, descriptivo, ya que se va determinar el nivel de empleabilidad y dominio de los tics por parte de los docentes aplicados en el aprendizaje en una Universidad Privada de la Región La Libertad.

\section{Diseño de investigación:}

El diseño es no experimental, ya que las variables no se van a manipular (Hernandez et al, 2014); de corte transversal pues los datos se van a extraer en un determinado espacio y tiempo con el propósito de describir las variables y su relación durante el proceso de enseñanza aprendizaje. Sampieri (2003)

Las investigaciones correlacionales simples permiten describir la incidencia de la variable independiente con relación a la dependiente, obteniendo datos en un solo momento determinado.

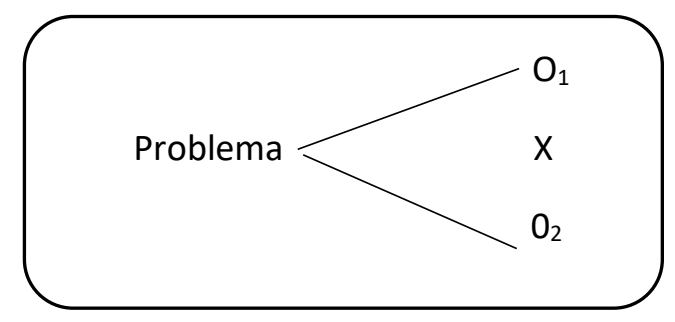


Técnicas de procesamiento de datos: Las tablas de distribución de frecuencias (absoluta y la porcentual) con las que se procesarán los ítems de los cuestionarios de encuesta.

La rho de Spearman y la Prueba t para la contratación de hipótesis.

\section{RESULTADOS Y DISCUSIÓN}

Tabla 1 Nivel de uso de las Herramientas Tic de los estudiantes de Derecho de una universidad privada de Trujillo, 2021.

\begin{tabular}{|l|c|c|}
\hline & Frecuencia & Porcentaje \\
\hline Regular & 3 & 3.1 \\
\hline Bueno & 39 & 40.2 \\
\hline Excelente & 55 & 56.7 \\
\hline Total & 97 & 100 \\
\hline
\end{tabular}

NOTA: Datos de las encuestas aplicadas a los estudiantes de I ciclo de la Escuela

Profesional de Derecho y Ciencias Políticas la Universidad Privada Antenor Orrego 2021.

\section{Anexo 4}

En la tabla 1, se puede percibir en relación a la variable TIC que existe en el nivel Excelente conformado por 55 estudiantes que equivale al 56.7\%; en el nivel Bueno conformado por 39 estudiantes que equivale al $40.2 \%$ y el nivel Regular conformado por 3 estudiantes que equivale al 3.1\%. En conclusión, el uso de las Herramientas Tic en una universidad privada de Trujillo es de nivel Bueno hacia el Excelente. Esto es el resultado de la interacción que se da desde el hogar, la escuela y la universidad, ya que todos los entes que rodean al educando juegan un rol protagónico en cierto momento durante el desarrollo de los estudiantes.

Tabla 2 Resultados obtenidos en la variable Aprendizaje en los estudiantes de Derecho de una universidad privada de Trujillo, 2021.

\begin{tabular}{|l|c|c|}
\hline & Frecuencia & Porcentaje \\
\hline Bueno & 22 & 22.7 \\
\hline Excelente & 74 & 76.3 \\
\hline Total & 96 & 99 \\
\hline Perdidos-Sistema & 1 & 1 \\
\hline Total & 97 & 100 \\
\hline
\end{tabular}

NOTA: Datos de las encuestas aplicadas a los estudiantes de I ciclo de la Escuela

Profesional de Derecho y Ciencias Políticas la Universidad Privada Antenor Orrego 2021. Anexo 7 
En la Tabla 2, se puede percibir en relación a la variable Aprendizaje los siguientes resultados: En el nivel Excelente conformado por 74 estudiantes que equivale al $76.3 \%$ y en el nivel Bueno conformado por 22 estudiantes que equivale al 22.7\%. En conclusión, el nivel de aprendizaje en los estudiantes de una universidad privada de Trujillo va del bueno al excelente. Como se puede apreciar el aprendizaje a través de los diferentes medios o herramientas que se pueden encontrar en la tecnología reflejan un incremento cognitivo en los estudiantes, a tal punto de modificar su panorama de desenvolvimiento académico.

\section{Tabla 3}

Resultados obtenidos a nivel de las dimensiones de la variable Aprendizaje en los estudiantes de Derecho de una universidad privada de Trujillo, 2021.

\begin{tabular}{|c|c|c|c|c|c|c|c|c|}
\hline \multirow[t]{3}{*}{ Dimensiones } & \multicolumn{8}{|c|}{ Aprendizaje } \\
\hline & \multicolumn{2}{|c|}{ Lectura y comunicación } & \multicolumn{2}{|c|}{$\begin{array}{l}\text { Morfología y } \\
\text { acentuación }\end{array}$} & \multicolumn{2}{|c|}{$\begin{array}{l}\text { Puntuación y el } \\
\text { párrafo }\end{array}$} & \multicolumn{2}{|c|}{\begin{tabular}{|l} 
Elinforme y la \\
exposición
\end{tabular}} \\
\hline & $\begin{array}{l}\text { Frecuen- } \\
\text { cia }\end{array}$ & $\begin{array}{l}\text { Porcen- } \\
\text { taje }\end{array}$ & $\begin{array}{l}\text { Frecuen- } \\
\text { cia } \\
\end{array}$ & $\begin{array}{l}\text { Porcen- } \\
\text { taje }\end{array}$ & $\begin{array}{l}\text { Frecuen- } \\
\text { cia } \\
\end{array}$ & $\begin{array}{l}\text { Porcen- } \\
\text { taje }\end{array}$ & $\begin{array}{l}\text { Frecuen- } \\
\text { cia }\end{array}$ & $\begin{array}{l}\text { Porcen- } \\
\text { taje }\end{array}$ \\
\hline Malo & - & - & - & . & - & - & - & - \\
\hline Regular & 4 & 4.1 & 4 & 4.1 & 1 & 1,0 & 2 & 2.1 \\
\hline Bueno & 24 & 24.7 & 31 & 32 & 27 & 27,8 & 28 & 28.9 \\
\hline Excelente & 69 & 71.1 & 62 & 63.9 & 69 & 71,1 & 67 & 69.1 \\
\hline Total & 97 & 100 & 97 & 100 & 97 & 100,0 & 97 & 100 \\
\hline
\end{tabular}

NOTA: Datos de las encuestas aplicadas a los estudiantes de I ciclo de la Escuela

Profesional de Derecho y Ciencias Políticas la Universidad Privada Antenor Orrego 2021.

\section{Anexo 5}

En la Tabla 3, se puede observar en relación a la dimensión 1: Lectura y Comunicación los siguientes resultados: en el nivel Excelente conformado por 69 estudiantes que equivale al $71.1 \%$, en el nivel Bueno conformado por 24 estudiantes que equivale al $24.7 \%$ y en el nivel Regular conformado por 4 estudiantes que equivale al 4.1\%. En cuanto a la dimensión 2: se puede observar en relación: Morfología y Acentuación los siguientes resultados: en el nivel Excelente conformado por 62 estudiantes que equivale al $63.9 \%$, en el nivel Bueno conformado por 31 estudiantes que equivale al 32\% y en el nivel Regular conformado por 4 estudiantes que equivale al 4.1\%. Así mismo, en relación a la dimensión 3: se puede observar en relación a Puntuación y el Párrafo los siguientes resultados: en el nivel Excelente 
conformado por 69 estudiantes que equivale al 71,1\%, en el nivel Bueno conformado por 27 estudiantes que equivale al 27,8\% y en el nivel Regular conformado por 1 estudiantes que equivale al1,0\%. Por último, en relación a la dimensión 4: El Informe y la Exposición los siguientes resultados: en el nivel Excelente conformado por 67 estudiantes que equivale al $69.1 \%$, en el nivel Bueno conformado por 28 estudiantes que equivale al $28.9 \%$ y en el nivel Regular conformado por 2 estudiantes que equivale al 2.1\%. En conclusión, de acuerdo a las dimensiones del Aprendizaje y los valores que se pueden apreciar afirmamos que tanto el docente como los estudiantes mientras más realicen el análisis y empleen las diversas herramientas que brinda la tecnología se verá un incremento significante en los estudiantes y por parte de los docentes la aplicación de estrategias generará un cambio radical en cuanto a la asimilación de contenidos y el procesamiento de la información, así como el manejo de todo este panorama cibernético.

En relación a los resultados obtenidos en la Tabla 1 de la encuesta Herramientas Tics aplicadas a los estudiantes nos permite ver como resultado de incidencia en el nivel excelente de un $56.7 \%$ este resultado es respaldado por Zambrano y Zambrano (2019) en su Tesis Las tecnologías de la información y las comunicaciones (TICs) en la educación superior: consideraciones teóricas realizada en la Universidad Laica Eloy Alfaro de Manabí, Ecuador sobre la empleabilidad de las Herramientas Tics; afirma que el empleo de estos recursos en el contexto universitario son de suma importancia y se convierte en una herramienta la cual fusiona una serie de elementos, campos, programas o estrategias de las cuales se puede servir el docente para incrementar el panorama cognitivo de los estudiantes.

Así mismo Francis (2017) en su artículo científico The Effects Of Technology On Student Motivation And Engagement In Classroom-Based Learning. New England, New England: University of New England DUNE aplicado a una población de 348 estudiantes a través del método de análisis de datos, expresa que la tecnología ha generado una creciente integración, desde el punto de vista de contextos de empleabilidad, y por la cantidad de información que se puede encontrar dentro de ella, generando un panorama de inclusión social.

Otro aspecto basado en la investigación está en relación a la Tabla 4 que nos muestra los resultados de la variable Aprendizaje con un valor de 76.3\%, Esto es respaldado por Rejas (2019). En su Tesis Aplicación de herramientas tecnológicas para la enseñanza - 
aprendizaje de los estudiantes de la Facultad de Administración de la Universidad Luis Gonzaga de Ica para optar el título de segunda especialidad profesional de tecnologías de información y comunicación arriba a la conclusión, afirmando que la influencia de las Tics en el desenvolvimiento académico de los estudiantes.es sustancias, pero está condiciona a la temática y a la forma de analizar y la estructuración de un determinado esquema de simplificación encontrados en la tecnología, generará un mayor nivel del aprendizaje se contrasta con el valor del coeficiente de correlación de Pearson $r=0,524$.

También lo ratifica Valarezo y Santos (2019) al describir en su artículo científico Las tecnologías del aprendizaje y el conocimiento en la formación docente las diversas formas de entender lo que se conoce como TAC O TIC que siendo el mismo panorama son herramientas didácticas a las cuales se debe recurrir o emplear durante el proceso de aprendizaje, ya que hacen factible la modificación del conocimiento tanto a nivel de los docentes como de los estudiantes.

\section{CONCLUSIONES}

El uso de las Herramientas Tic en el desarrollo académico es preponderante en los estudiantes, ya que una vez realizado el procesamiento estadístico se puede percibir en la Tabla 1un nivel de excelente con un $56.7 \%$ reflejando una fuerte incidencia; esto está condicionado al criterio del docente pues debe considerar la temática a desarrollar y emplear el recurso o herramienta con mayor efectividad para beneficio de los estudiantes, ya que puede encontrar diferentes campos con información detallada o herramientas a emplear al momento de realizar el análisis de una determinada temática.

El manejo de todos estos recursos cibernéticos aplicados a la enseñanza en todos los niveles generan un incremento positivo tanto en el docente, pero con mayor relevancia en la asimilación de contenidos por parte de los estudiantes ya que con respecto a la variable Aprendizaje, Tabla 4 se puede percibir un valor de $76.3 \%$, esto sumado a la retroalimentación en los hogares por parte de los padres se puede afirmar que todo el contexto estudiantil llegará a incrementar con mayor efectividad su asimilación de contenidos.

El desenvolvimiento del docente y estudiante debe estar enmarcado, en la actualidad, en el contexto cibernético, ya que este nos brinda una serie de recursos, programas, estructuras los cuales contribuyen a desarrollar el panorama cognitivo de ambos. En este panorama nos 
encontramos con la dimensión Internet la cual refleja un nivel excelente con un valor de $70.1 \%$ (Tabla 2) de empleabilidad por parte de los estudiantes. También encontramos con la dimensión Foro que presenta un nivel de bueno con un valor de 43.3\% (Tabla 3). Al ver estos porcentajes el docente debe incidir con mayor frecuencia en su empleabilidad desarrollando estrategias apoyado en las diferentes herramientas que le brinda la cibernética, generando mayor interacción entre otros estudiantes, la aplicación y retroalimentación de su conocimiento para desarrollar sus habilidades estudiantiles.

Dentro del campo del Aprendizaje en el contexto de los estudiantes de una universidad privada de Trujillo y de acuerdo a su programación visualizamos las siguientes realidades en la Tabla 6: en cuanto a Lectura y comunicación un valor de $71.1 \%$, en Morfología y acentuación un valor de 63.9\%, en Puntuación y el párrafo un valor de $71.1 \%$ y en el Informe y la exposición un valor de $69.1 \%$. Estos valores nos permiten ver la efectividad del empleo de los diferentes campos de la cibernética para los estudiantes.

\section{LISTA DE REFERENCIAS}

Arias, J. (2019). Uso del Exelearning, aplicación de contenidos digitales y su relación con el proceso aprendizaje. Huacho - Lima: Universidad Nacional José Faustino Sánchez Carrión.

Ávila, C. D. (2016). La inclusión de las TIC como desafío. Universidad Siglo XXI, pp.51.

Barredas, F. (1994). Sobre el conductismo en B. Skinner, \& S. Martinez Roca (Ed.), sobre el conductismo (F. Barrera, Trad., Martinez Roca, S.A ed., págs. 14 - 18). Buenos Aires - Argentina: Editorial Planeta Argentina, S.A.I.C.

Basri, W., Alandejani, J., \& Almadani, F. (2018). ICT adoption Impact on students Academic Performance: Evidence from Saudi Universities. Education Research International, 9.

Belando-Montoro, María (2017). Aprendizaje a lo largo de la vida: concepto y componentes. Artículo monográfico. Pedagogía Escolar.Universidad Complutense de Madrid. España.Vol.75.

Beyneto-Seoane, M. C.-S. (2018). Análisis de la actual formación docente en competencias tic. por una nueva perspectiva basada en las competencias, las experiencias y los 
conocimientos previos de los docentes. Profesorado. Revista de Curriculum y formación del profesorado, 22(4), 91-110. doi:10.30827/ profesorado. .v22i4.8396

Briceño, L., Flores, R., \& Gómez, D. (2019). Usos de las Tics en preescolar: hacia la integración curricular. Panorama, Vol. 13 - 24.

Cabrera, E. (2019). Entornos virtuales de aprendizaje mediante una nueva metodología aplicados a las competencias tecnológicas del Docente. Lima - Perú: Universidad de San Martin de Porres.

Candia, M. (2018). Incursión de las TIC en la educación superior. Paraguay: iberoamérica divulga. Pág. 1 https://www.oei.es/historico/divulgacioncientifica/?Incursion-de-las$\underline{\text { TIC-en-la-educacion-superior }}$

Carrasco, J. (2019). Las TIC y su influencia en la calidad del aprendizaje universitario. Revista Industrial, 22(1), 1.

Castro Mendez, N., Suárez Cretton, X., \& Soto Espinoza, V. (2016). El uso del foro virtual para desarrollar el aprendizaje autorregulado de los estudiantes universitarios. (I. P. Nacional, Ed.) Innovación Educativa, 16(70), 23-41.

Castro, I. (2017). La Exposición como Estrategia de Aprendizaje y Evaluación en el Aula. En I. Castro, \& U. d. Hemisferios (Ed.), La Exposición como Estrategia de Aprendizaje y Evaluación en el Aula (págs. 9 - 10). Quito, Pichincha , Ecuador: Sello Editorial Razón y palabra.

Ccoillo, M. (2018). Ranking de Sunedu revela las mejores universidades del Perú. La República, pág. 1.

Cejas, R. (2018). La formación en TIC del profesorado y su transferencia a la función docente. Barcelona, España: Universitat Autónoma de Barcelona.

Daniels, H. (2003). Vygotsky y la pedagogía. En H. Daniels, T. p. por, \& P. SAICF (Ed.), Vygotsky y la pedagogía (G. Barberán, Trad., pág. 275). Barcelona, España: Paidós Iberica S.A.

De La Cruz, J., \& Rodríguez, E. (2019). La investigación: más allá del ranking de las universidades. (U. R. Facultad de Medicina Humana, Ed.) Editorial. Rev. Fac. Med. Hum, 11. 
Desarrollo, R. R. (2017). La implicación de las TIC en la educación: Alcances, Limitaciones y Prospectiva. RIDE. Revista Iberoamericana para la Investigación y Desarrollo, $8(15)$.

Edoru, J., \& Sanni, T. (2019). Information and communication technology in ugandan higher education. chapter four eduru edited, 8 .

Ezcurra, A., Bariola, N., Naters, L., \& Romero, L. (2007). Iniciarse en la redacción universitaria: exámenes, trabajos y reseñas. En A. Ezcurra, N. Bariola, L. Naters, \& L. Romero, Iniciarse en la redacción universitaria: exámenes, trabajos y reseñas (Vol. 2, págs. 63 - 64). Lima, Lima, Perú: Fondo Editorial Universidad Catolica del Perú.

Figueras, C. (2014). Pragmática de la Puntuación y nuevas tecnologías. (C. Figueras, Ed.) Revista Norma (4), 135-160.

Flórez R., Rita; Castro M., Jaime Alberto; Arias V., Nicolas; [et al] (2016). Aprendizaje, cognición y mediaciones en la escuela Una mirada desde la investigación en instituciones educativas del Distrito Capital. Texto. Bogota-Colombia

Francis, J. (2017). The Effects Of Technology On Student Motivation And Engagement In Classroom-Based Learning. New England, New England: University of New England DUNE: DigitalUNE.

García de Merlano, M. O. (2013). TIC, educación y sociedad. reflexiones y estudio de caso a nivel iberoamericano (Vol. 2). Bogotá, Colombia: Corporación Colombiana Digital. Obtenido de www.colombiadigital.net

García, M., Reyes, J., \& Godinez, G. (2017). Las Tic en la educación superior, innovaciones y retos. Iberoamericana de las Ciencias Sociales y Humanísticas, 6(12), 18.

Gil, E. (2002). Identidad y Nuevas tecnologías. Obtenido de http:www.voc.edu/ web/esplart/gil0902/htrr

Gonzales, J. (2004). Estudio sobre el párrafo. (L. G. Universidad de Alicante. Departamento de Filología Española, Ed.) Revista - ELUA(18), 87 - 106.

Gros, B. (2011). Evolución y retos de la educación virtual. construyendo el e-learning del siglo XXI (Vol. I edición). (B. GROS, Ed.) Barcelona, España: Editorial UOC. Obtenido de http://www.editorialuoc.com/ 
Hermosa del Vasto, P. M. (2015). Influencias de las tecnologías de información y comunicación (TIC) en el proceso enseñanza - aprendizaje: una mejora de las competencias digitales. Revista Científica General José María Cordova, 121 - 132.

Hernández Sampieri, R. F. (2010). Metodología de la investigación (quinta ed.). D.F. México: McGraw-Hill.

Hernández Sampieri, R., \& Fernández Collado, C. y. (2014). Metodología de la Investigación (sexta ed.). D.F. México: McGraw-Hill/Interamericana Editores, S.A. DE C.V. doi:ISBN: 978-1-4562-2396-0

Hernández, B. (s/f). Ortografía y Redacción para todos. En B. Hernández, Ortografía y Redacción para todos (pág. 71).

Hernández, M. (2017). Impacto de las TIC en la educación: Retos y Perspectivas. Propósitos y Representaciones, 5(1), 334.

Hinojo, F., Aznar, I., Cáceres, M., \& Romero, J. (2019). Artificial Intelligence in Higher Education: A Bibliometric Study on its Impact in theScientific Literature. education sciences MDPI, 8.

Instituto Nacional de Estadística e Informática. (2018). Estadísticas de las Tecnologías de Información y Comunicación en los Hogares. LIMA: INEI.

IPEBA. (2013). Comunicación: Comunicación oral. En Ipeba, \& M. d. Educación (Ed.), Comunicación: Comunicación oral (págs. 7 - 9). Lima: Centro de Producción Editorial e Imprenta de la Universidad Nacional Mayor de San Marcos.

Islas, C. (2017). La implicación de las TIC en la educación: Alcances, Limitaciiones y Prospectiva. Revista Iberoamericana para la Investigación y el Desarrollo Educativo, $8(15), 16$.

Kutugata Estrada, A. (2016). Foros de discusión: herramienta para incrementar el pensamiento critico en la educación superior. Scielo, 8(2). doi:ISSN 2007-1094

Marcos Oswaldo Arnao Vásquez, C. E. (2016). Lectura y escritura con recursos TIC en la educación Superior. Innoeduca. International Journal of Techlology and Educational Innovation, p. 71.

Mateus, J. (2018). Internet como derecho constitucional en el Perú: sentido y desafío. Eduacared, pág. 1. 
Medina, D. (2018). El rol de las universidades peruanas frente a la investigación y el desarrollo tecnológico. Propósitos y Representaciones, 6(2), 7171 - 718.

Modelo Educativo Crítico con Enfoque de Competencias. (2017). En M. A. Gutierrez, Modelo educativo crítico con enfoque de competencias (pág. 124). Colombia: Ediciones Universidad Cooperativa de Colombia - GREEN PAPERS.

Moh'd, A. (2017). The Impact Of Using Social Media And Internet On Academi Performance: Case Study Bahrain Unviersities. (A. Moh’d, Ed.) Ahlia University, 1. Monsalve, J. (2018). Influencia del uso de las Tics en la evaluación del desempeño del docente en el sistema educativo universitario de Lima. Lima - Perú: UNMSM.

Moyazzem, M. (2019). Impact of Mobile Phone Usage on Academic. World Scientific News, 164.

Ochoa, X. y. (2002). http://www.ruv.itesm.mx/especiales/citela/documentos /material/ módulos/módulos2/contenidoii.html.

Pinto, A. (2017). El sistema de comunicación y su incidencia en la gestión de la investigación desarrollada en la Universidad Técnica de Babahoyo, provincia de los Ríos - Ecuador. Provincia de los Ríos - Ecuador: Universidad Nacional Mayor de San Marcos.

Ramírez, O. (2019). el acento ortográfico y sus reglas. (O. Ramírez, Ed.) Aboutespañol, 1.

Regueiro, M., Delgado, I., Lazaro, F., \& Boneroso, L. (2019). Proyecto de Innovación Plataforma gramatical de enseñanza de español como lengua extrajera. Universidad Complutense de Madrid, 1.

Rejas, J. (2019). Aplicación de herramientas tecnológicas para la enseñanza - aprendizaje de los estudiantes de la Facultad de Administración de la Universidad Luis Gonzaga de Ica 2016-2017. Huancavelica.

Reyes, M. E. (2017). Modelo de calidad aviso en la implementación de entornos Blended Learning para la mejora del proceso enseñanza - aprendizaje en la educación superior universitaria. Lambayaque - Chiclayo.

Reyes, N. S. (2017). Propuesta metodológica para el aprendizaje de inglés en la Universidad Tecnológica Equinoccial (Ecuador) con el uso de las Tic. Universidad de Extremadura. 
Rodríguez, J. (2017). El construccionismo como modelo pedagogico para el uso de las Tics en la educación. 25 - 26.

Salgado Reyes, N. (2017). Propues metodológica para el aprendezaje de inglés en la Universidad Tecnológica Equinoccial (Ecuador) con el uso de la TIC. Quito Ecuador.

Sanabria, M. (2013). La teoría de las inteligencias múltiples desde la perspectiva del asesoramiento psicopedagógico en el contexto educativo. (Redalyc, Ed.) Revista ESPIGA, XII (25), 37-38.

Selwin, N. (2014). The Internet and Education. Open Mind, 1.

Spante, M., Sofkova, S., Lundin, M., \& Algers, A. (2018). Digital competence and digital literacy in higher education research: Systematic review of concept use. (T. $U$. Shuyan Wang, Ed.) cogent education, 5(1519143), 1.t. (s.f.).

Taylor, B., \& Cantwell, B. 1. (2018). Unequal Higher Education in the United States: Unequal Higher Education in the United States. social sciences, 20.

Thompson, P. G. (2012). Administración estrategíca. teoría y casos. En P. G. Thompson, \& I. e. S.A. (Ed.), Administración estrategíca. teoría y casos (décimaoctava ed.). Mexico: McGraw. Hill/ Educación. doi:ISBN 978-970-10-6402-3

Tirado, P., \& Roque, M. d. (2019). TIC y Contextos Educativos: Frecuencia de uso y función por universitarios. Edutec. Revista Electrónica de Tecnología Educativa (67), 43.

UNESCO. (2017). The International Forum on ICT and Education 2030. Seize Digital opportunities, lead education transformation (pág. 1). Qingdao, the People's Republic of China: UNESCO.

Universia. (2018). 10 tips para hacer una presentación oral exitosa. En u. colombia, 10 tips para hacer una presentación oral exitosa (pág. 1). colombia, Colombia: universia colombia.

University of Glasgow. (2019). The Word University Rankings. World Changing Glasgow, pp. $1-25$.

Valarezo, J., \& Santos, O. (MAYO de 2019). Las tecnologías del aprendizaje y el conocimiento en la formación docente. Revista Conrado, 15(68), 185. 
Valle, Y. (2018). Sin las TIC, la educación superior y de posgrado no está completa. En Y. Valle, Sin las TIC, la educación superior y de posgrado no está completa (pág. 1). Lima - Perú: ESAN.

Venegas-Ramos, L., Luzardo Martínez, H. J., \& Pereira Santana, A. (2020). Conocimiento, formación y uso de herramientas TIC aplicadas a la Educación Superior por el profesorado de la Universidad Miguel de Cervantes. Edutec. Revista Electrónica De Tecnología Educativa, (71), 35-52. https://doi.org/10.21556/edutec.2020.71.1405

Vidal, J., Rui, P., \& Rocha, A. (30 de julio de 2019). A systematic literature review on maturity models for information systems in higher education institutions. Routledge Taylor \& Fracis Group, 1.

Villarroel, V. A. (2017). Competencias Pedagógicas que Caracterizan a un Docente Universitario de Excelencia: Un Estudio de Caso que Incorpora la Perspectiva de Docentes y Estudiantes. Form. Univ. [online]-Scielo, 10(4), 75-96. doi:10.4067/S0718-50062017000400008

Wazarus, I. (2019). Morfología de la no-narrativa en la animación. Universitat Politécnica de Valencia, 9, 1.

Yeravdekar, V., Ghosh, R., \& Mishra, S. (octubre de 2018). University of the Future. FICCI, 52.

Zambrano, D., \& Zambrano, M. (2019). Las tecnologías de la información y las comunicaciones (TICs) en la educación superior: consideraciones teóricas. Revista Electrónica Formación y Calidad Educativa (REFCalE) ISSN 1390-9010 Ecuador, p. 226. 